\title{
Intravenous Immunoglobulin in BK Virus Nephropathy
}

\author{
Elizabeth I. Anyaegbu*,1 and Stanley P. Hmiel² \\ ${ }^{I}$ Driscoll Children's Hospital, Kidney Center, Corpus Christi, Texas, USA \\ ${ }^{2}$ Washington University School of Medicine, Division of Pediatric Nephrology, St Louis, Missouri, USA
}

\begin{abstract}
The incidence of post transplant viral infections has increased with the use of more potent immunosuppressive regimens. Consequently, BK virus nephropathy $(\mathrm{BKVN})$ has arisen as a significant cause of graft dysfunction and loss. Reduction of immunosuppression is the first line management of post-transplant viral infections. Other therapies such as intravenous immunoglobulin (IVIg), cidofovir, leflunomide and fluoroquinolones have been tried with varying degrees of success.

We report our experience with IVIg in three pediatric renal transplant recipients who presented with allograft dysfunction. First, we describe two cases of biopsy proven BKVN, one diagnosed with undetectable viral titers in plasma, demonstrating that BKVN can occur even at low viral loads. We also present a pediatric renal transplant recipient with persistent BK viremia and allograft dysfunction who responded to therapy with recovery of renal function and clearance of viremia. Therefore we conclude that IVIg is efficacious in the treatment of persistent BK viremia and BKVN. The appropriate dose, frequency and duration of therapy require further study.
\end{abstract}

Keywords: Allograft dysfunction, BK viremia, BK virus nephropathy (BKVN), immunosuppression reduction, intravenous immunoglobulin (IVIg), pediatric transplantation.

\section{INTRODUCTION}

BK virus nephropathy (BKVN) has become of significant concern as a cause of graft dysfunction and loss in the renal transplant population. Dormant BK virus is reactivated in the setting of increased immunosuppression, causing tubulointerstitial nephritis in up to $8 \%$ of patients [1]. Presently no standard therapy for BK viremia and nephropathy has been established.

Reduction in immunosuppression is accepted as the first line therapy in the management of BKVN [2]. The use of leflunomide, cidofovir, IVIg and fluoquinolones as adjunct therapy in addition to immunosuppression reduction in the management of BK virus nephropathy has been evaluated in several small clinical studies [3-6]. There have however been no prospective randomized studies to compare the various agents and provide much needed information on long-term benefits.

Human intravenous immunoglobulin has immunomodulatory properties and has been used successfully for transplant related indications including treatment of antibody mediated rejection; desensitization of highly sensitized transplant recipients and in treatment of post-transplant infectious complications like parvovirus B19, cytomegalovirus and BKVN [7].

\footnotetext{
*Address correspondence to this author at the University of Texas Medical Branch, Pediatric Nephrology, Driscoll Children's Hospital Kidney Center, 3533 South Alameda Street, Corpus Christi, Texas, USA;

Tel: (361) 694-6732; Fax: (361) 694-6859;

E-mail: Elizabeth.Anyaegbu@dchstx.org
}

One of the authors, Anyaegbu et al., has previously reported her experience using IVIg for treatment of persistent BK viremia and BK nephropathy [4]. There have been conflicting reports on the efficacy of IVIg in the treatment of BKVN. Majority of the reports have described successful use in the management of BKVN. However there have been reports of an increase in BK viral load following IVIg therapy $[8,9]$.

We conducted a retrospective review at the Washington University in St Louis of pediatric renal transplant recipients who received IVIg therapy for management of BKVN and report our successful treatment of BK viremia and nephropathy with immunosuppression reduction and administration of IVIg. Patients were screened for BK virus when they presented with elevations in serum creatinine. This was before the implementation of our prospective screening protocol for BK viriuria and viremia. Our first line strategy for management of BK viremia was immunosuppression reduction, which was sufficient therapy in the majority of patients. Three patients were diagnosed with BKVN and received IVIg following an inadequate response to only immunosuppression reduction. The therapeutic benefit of IVIg after an insufficient response to immunosuppression reduction was demonstrated in these patients. Our report shows that administration of IVIg is an effective option for the management of BKVN.

\section{CASE 1}

A 15-year-old Caucasian male with end stage renal disease secondary to complications of William's syndrome received a renal transplant from a cadaveric donor. At the 
time of transplantation both the donor and recipient were positive for cytomegalovirus. Induction agents included 5 doses of rabbit Thymoglobulin and pulse steroids that were rapidly tapered. There was excellent graft function with rapid decline in serum creatinine. He was maintained on a steroid free regimen consisting of tacrolimus and mycophenolate mofetil. Ureteral stents were removed 1 month after transplant and graft function remained stable at a baseline of $0.7-0.9 \mathrm{mg} / \mathrm{dl}$.

Two years post-transplant, his serum creatinine increased to $1.5 \mathrm{mg} / \mathrm{dl}$. BK viremia was confirmed by quantitative detection of BK virus in plasma. BK viral titers in urine and plasma at diagnosis were greater than $1 \times 10^{6}$ copies and $1.39 \times 10^{6}$ respectively. An allograft biopsy showed diffuse tubulointerstitial inflammation and a positive stain for Simian virus 40 (SV-40) large T-antigen. C4d staining was negative. His mycophenolate mofetil was discontinued and his tacrolimus dose was reduced to $1 \mathrm{mg}$ twice a day targeting tacrolimus troughs of $3-4 \mathrm{ng} / \mathrm{ml}$. There was an inadequate response to reduction in immunosuppression, which prompted administration of IVIg. He received 6 doses of IVIg $(0.5 \mathrm{~g} / \mathrm{kg})$ monthly with clearance of viremia and improvement in allograft function.

\section{CASE 2}

A second patient was diagnosed with BKVN by biopsy. He was found to have nephropathy in the setting of only BK viruria and negative $\mathrm{BK}$ in plasma 77 days post transplant. Urine viral titers were $3.23 \times 10^{6}$ with undetectable BK titers in plasma. He received a heart transplant as an infant and subsequently developed end stage renal disease from cyclosporine nephrotoxicity. He received a living related kidney from his mother. Induction immunosuppression consisted of rabbit Thymoglobulin $1.5 \mathrm{mg} / \mathrm{kg} /$ dose given daily for a total of 5 doses and pulse intravenous methylprednisolone for 4 days. This was followed by maintenance immunosuppression with mycophenolate mofetil and tacrolimus.

During a routine post-transplant follow-up visit, his serum creatinine was found to have increased to $0.8 \mathrm{mg} / \mathrm{dl}$ from a baseline of $0.4 \mathrm{mg} / \mathrm{dl}$, prompting a biopsy that showed a positive SV-40 stain. Immunosuppression was reduced by withdrawal of mycophenolate mofetil and reduction in the dose of tacrolimus to target trough levels of $4-5 \mathrm{ng} / \mathrm{ml}$.

Viral clearance and improvement in graft function was achieved with reduction in immunosuppression and monthly IVIg $(0.5 \mathrm{~g} / \mathrm{kg})$ therapy for 6 months. His graft function however did not return to his previous baseline.

\section{CASE 3}

A 16-year-old Hispanic male who was an illegal immigrant received a cadaveric kidney transplant due to end stage renal disease secondary to nephronopthisis. Graft function was excellent following induction with rabbit Thymoglobulin and pulse steroid therapy. $\mathrm{He}$ was maintained on a steroid free regimen consisting of tacrolimus and mycophenolate mofetil. He developed acute rejection due to missed doses of immunosuppressive medications that resulted from insufficient funds to purchase medications. He was treated with Thymoglobulin and pulse steroid therapy with recovery of function to his previous baseline of 0.9 $\mathrm{mg} / \mathrm{dl}$.

A few months later, he was found to have an elevation in his serum creatinine. His serum creatinine peaked at 2.2 $\mathrm{mg} / \mathrm{dl}$. BK viremia was detected and his immunosuppression was reduced. Initially the dose of mycophenolate mofetil was reduced by $50 \%$ and with persistent viremia was discontinued. The next step was a staged reduction in tacrolimus dosing to target troughs of $4-5 \mathrm{ng} / \mathrm{ml}$. With persistent viremia, he received IVIg. Viral clearance was seen after 5 monthly doses of $0.5 \mathrm{~g} / \mathrm{kg}$ with stabilization of graft function and a decrease in serum creatinine to $1 \mathrm{mg} / \mathrm{dl}$.

\section{DISCUSSION}

Pediatric renal transplant recipients are at high risk for BK virus infection post transplant because they are more likely to be naïve to the BK virus. Other risk factors include HLA mismatch, the intensity of immunosuppression with an increased incidence following treatment of rejection with intensified immunosuppression using lymphocyte-depleting therapy [10].

Prospective screening protocols have led to earlier detection of BK viremia, which has helped minimize the effect of BKVN on graft survival. Nonetheless, with detection of BK viruria and viremia, there is insufficient evidence to support additional therapy if there is an inadequate response to reduction in immunosuppressive therapy or in the face of established nephropathy.

Reduction in immunosuppression is accepted as the firstline management of BK viremia and nephropathy [2]. It has been used as the sole therapy for BK viremia and nephropathy [11]. The strategy for targeted reduction in immunosuppression varies among transplant centers. The most common approach involves decreasing and then discontinuation of the anti-proliferative agent. The calcineurin inhibitor is then decreased if viremia persists. In a cohort study, Schaub et al. outlined their reduction protocol, which initially involves reduction in calcineurin therapy by targeting lower trough levels. This was done by setting a lower tacrolimus trough than would be expected for the post-transplant period i.e. targeting a trough of $6-8 \mathrm{ng} / \mathrm{ml}$ instead of a trough of $8-10 \mathrm{ng} / \mathrm{ml}$. If viremia persisted, the calcineurin inhibitor was further reduced (eg the tacrolimus trough levels were decreased from 6-8 to $4-6 \mathrm{ng} / \mathrm{ml}$ ). The final stage in the protocol was a sequential reduction in mycophenolate moefetil dosing in increments of $50 \%$. Using this staged reduction protocol, viral clearance and stabilization of graft function was seen with immunosuppression reduction alone [11]. Reduction in immunosuppression is however associated with an increased incidence of acute rejection which has been reported in up to $25 \%$ of cases [12].

Reports have described the use of agents like cidofovir, leflunomide, fluoroquinolones and IVIg with varied success $[3-5,13,14]$. Cidofovir has anti-polyoma activities, which has been demonstrated in in vitro experiments. It is however extremely nephrotoxic which limits its use for management of BKVN. It has also been associated with a recurrence of $\mathrm{BK}$ viremia and persistent $\mathrm{BKVN}$ after an initial response 
[6]. Leflunomide has anti-viral properties against BK virus and is an attractive option for treatment of BKVN since it has minimal nephrotoxicity. It has been used successfully in adult and pediatric transplant recipients in combination with immunosuppression reduction [3, 13]. Fluoroquinolones inhibits polyoma virus replication in vitro and has been shown to decrease BK viral load. It has been used as a prophylactic agent. Garbardi et al. demonstrated a significantly lower incidence of BK viremia at 1 year in a cohort of 160 patients who received fluoroquinolone therapy for prophylaxis for at least 1 month post-transplant [5].

Case reports have emerged that have documented an increase in BK viral load following administration of IVIg. Boonyapredee $\mathrm{M}$ et al, reported their experience with a 57year old female renal transplant recipient who underwent a biopsy for allograft dysfunction in the setting of BK viremia 8 weeks post-transplant. The histological findings were consistent with Banff $1 \mathrm{~b}$ acute cellular rejection and a negative stain for SV-40 and negative C4d staining of peritubular capillaries. The patient was given $2 \mathrm{~g} / \mathrm{kg}$ of IVIg with no adjustments to her baseline immunosuppression since there was inadequate evidence to make a diagnosis of BKVN. Her BK viral load increased exponentially after administration of IVIg with a further increase in her serum creatinine. A repeat biopsy revealed a positive SV-40 stain within the epithelial cells of the tubules and moderate tubulitis. Her immunosuppression was promptly decreased and she received leflunomide for treatment of BKVN and a steroid pulse for treatment of concurrent acute cellular rejection. Her BK viral load decreased subsequently and there was improvement in her graft function reported [8].

This finding is similar to a report by Maggiore et al. who describes the case of a 49-year old woman who underwent a renal biopsy when she presented with an elevation in her serum creatinine up to $2.4 \mathrm{mg} / \mathrm{dl}$ from a baseline of $1.6-1.8$ $\mathrm{mg} / \mathrm{dl} 14$ months post-transplant. She was found to have BKVN and immunosuppression was reduced and she received $2 \mathrm{~g} / \mathrm{kg}$ of IVIg over 5 days. Her BK viral load was closely monitored and observed to increase in the first week following IVIg therapy from 7720 copies/ml to a peak of 144,440-copies/ ml after IVIg administration before a progressive decline was seen over a 12 -week period. Her graft function showed gradual improvement with a decrease in her serum creatinine to a new baseline of $2 \mathrm{mg} / \mathrm{dl}$ in the 29-month follow-up period [9].

IVIg has anti-inflammatory and BK neutralizing properties demonstrated in in vitro experiments [15]. It has been used for treatment of BKVN following an inadequate response to reduction in immunosuppression and has been reported to lead to a stabilization of allograft function, clearance of viremia and histological resolution of BKVN [4, 14].

Reports exist on adult and pediatric transplant recipients with BK viremia and nephropathy where IVIg was used with improvement in renal function and significant graft survival demonstrated. There was similarity in the dosing strategies for IVIg in the reports of the largest adult and pediatric cohorts $[4,14]$. There reports provided some insight into the utility of IVIg combined with immunosuppression reduction without the confounding effects of other therapeutic agents. In the report by Sharma et al, stabilization of renal function and histological resolution of BKVN was seen with IVIg after recurrent BK viremia developed with discontinuation of cidofovir therapy [6].

We utilized low dose IVIg treatments $(0.5 \mathrm{~g} / \mathrm{kg})$ given monthly over a 5 to 6 month period, which was similar to the protocol described by Sharma et al. [6]. The most common side effect seen was post infusion headache, which was easily managed with acetaminophen. No case of acute kidney injury was seen in our cohort as a result of IVIg therapy. Our choice of low dose therapy distributed over a period of time was based on the regimen used to treat other post transplant viral infections. It was effective and led to better inhibition of viral replication and better overall viral eradication since viral clearance is usually slow in the face of immunosuppression. Using low dose therapy also helped to minimize the infusion related complications frequently seen with high dose therapy.

Our findings highlight the effectiveness of IVIg as second line therapy following a suboptimal response to immunosuppression reduction and offer an alternative therapy to the current management strategies. The optimal dosing, frequency and duration of therapy needs to be determined. Also, the indication for treatment, cost effectiveness, availability and side effect profile require further study.

The fact that the histological features of BKVN on light microscopy are often difficult to distinguish from acute cellular rejection and BKVN sometimes coexist with rejection makes IVIg an attractive alternative strategy. Its anti-BK attributes together with the anti-rejection effects make it a unique therapeutic option in protecting against rejection, which can occur following reduction of immunosuppression. It is also the ideal treatment when the diagnosis is unclear or while awaiting results of the SV-40 staining for the BK virus.

Despite being expensive and in limited supply, IVIg is an appealing therapeutic option for the rising threat of BK viremia and BKVN in the setting of less well-defined options. Further study is needed to compare its efficacy to the other management options and longer follow-up is required to evaluate long-term benefits and graft survival.

\section{ABBREVIATIONS}

$$
\begin{array}{ll}
\mathrm{BKVN} & =\mathrm{BK} \text { virus nephropathy } \\
\mathrm{IVIg} & =\text { Intravenous immunoglobulin }
\end{array}
$$

\section{CONFLICT OF INTEREST}

The authors confirm that this article content has no conflict of interest.

\section{ACKNOWLEDGEMENTS}

This project was supported by the Washington University in St Louis Institute of Clinical and Translational Sciences (ICTS) UL1 grant. 


\section{REFERENCES}

[1] Hirsch HH, Knowles W, Dickenmann M, et al. Prospective study of polyomavirus type BK replication and nephropathy in renaltransplant recipients. N Engl J Med 2002; 347(7): 488-96.

[2] Hirsch HH, Randhawa P. BK virus in solid organ transplant recipients. Am J Transplant 2009; 9 Suppl 4: S136-46.

[3] Araya CE, Garin EH, Neiberger RE, Dharnidharka VR. Leflunomide therapy for BK virus allograft nephropathy in pediatric and young adult kidney transplant recipients. Pediatric transplant 2010; 14(1): 145-50.

[4] Anyaegbu EI, Almond PS, Milligan T, Allen WR, Gharaybeh S, Al-Akash SI. Intravenous immunoglobulin therapy in the treatment of BK viremia and nephropathy in pediatric renal transplant recipients. Pediatric transplant 2012; 16(1): E19-24.

[5] Gabardi S, Waikar SS, Martin S, et al. Evaluation of fluoroquinolones for the prevention of $\mathrm{BK}$ viremia after renal transplantation. Clin J Am Soc Nephrol 2010; 5(7): 1298-304.

[6] Sharma AP, Moussa M, Casier S, Rehman F, Filler G, Grimmer J. Intravenous immunoglobulin as rescue therapy for BK virus nephropathy. Pediatric transplant 2009; 13(1): 123-9.

[7] Jordan SC, Toyoda M, Kahwaji J, Vo AA. Clinical aspects of intravenous immunoglobulin use in solid organ transplant recipients. Am J Trnsplant 2011; 11(2): 196-202.

[8] Boonyapredee M, Knight K, Little D. Increased BK viremia and progression to BK-virus nephropathy following high-dose intravenous immunoglobulin for acute cellular rejection. Mil Med 2014; 179(6): e699-702.

[9] Maggiore U, Medici MC, Vaglio A, Buzio C. Increased viral load after intravenous immunoglobulin therapy for BK virus-associated nephropathy. Transpl Infect Dis 2010; 12(5): 470-2.

[10] Prince O, Savic S, Dickenmann M, Steiger J, Bubendorf L, Mihatsch MJ. Risk factors for polyoma virus nephropathy. Nephrol Dial Transplant 2009; 24(3): 1024-33.

[11] Schaub S, Hirsch HH, Dickenmann M, et al. Reducing immunosuppression preserves allograft function in presumptive and definitive polyomavirus-associated nephropathy. Am J Transplant 2010; 10(12): 2615-23.

[12] Bohl DL, Brennan DC. BK virus nephropathy and kidney transplantation. Clin J Am Soc Nephrol 2007; 2 (Suppl 1): S36-46.

[13] Josephson MA, Gillen D, Javaid B, et al. Treatment of renal allograft polyoma BK virus infection with leflunomide. Transplantation 2006; 81(5): 704-10.

[14] Sener A, House AA, Jevnikar AM, et al. Intravenous immunoglobulin as a treatment for BK virus associated nephropathy: one-year follow-up of renal allograft recipients. Transplantation 2006; 81(1): 117-20.

[15] Randhawa PS, Schonder K, Shapiro R, Farasati N, Huang Y. Polyomavirus BK neutralizing activity in human immunoglobulin preparations. Transplantation 2010; 89(12): 1462-5.

(C) Anyaegbu and Hmiel.; Licensee Bentham Open.

This is an open access article licensed under the terms of the Creative Commons Attribution Non-Commercial License (http://creativecommons.org/licenses/by-nc/3.0/) which permits unrestricted, non-commercial use, distribution and reproduction in any medium, provided the work is properly cited. 